\title{
Quantum-critical spin dynamics in a Tomonaga-Luttinger liquid studied with muon-spin relaxation
}

\author{
J. S. Möller, ${ }^{1, *}$ T. Lancaster, ${ }^{2}$ S. J. Blundell, ${ }^{1}$ F. L. Pratt, ${ }^{3}$ P. J. Baker, ${ }^{3}$ F. Xiao, ${ }^{2}$ R. C. Williams, ${ }^{2}$ \\ W. Hayes, ${ }^{1}$ M. M. Turnbull, ${ }^{4}$ and C. P. Landee ${ }^{4}$ \\ ${ }^{1}$ Department of Physics, Clarendon Laboratory, Oxford University, Parks Road, Oxford OX1 3PU, United Kingdom \\ ${ }^{2}$ Centre for Materials Physics, Durham University, South Road, Durham DH1 3LE, United Kingdom \\ ${ }^{3}$ ISIS Facility, STFC Rutherford Appleton Laboratory, Didcot OX11 OQX, United Kingdom \\ ${ }^{4}$ Carlson School of Chemistry and Biochemistry and Department of Physics, Clark University, Worcester, Massachusetts 01610, USA
}

(Received 9 August 2016; published 3 January 2017)

\begin{abstract}
We demonstrate that quantum-critical spin dynamics can be probed in high magnetic fields using muon-spin relaxation $\left(\mu^{+} \mathrm{SR}\right)$. Our model system is the strong-leg spin ladder bis(2,3-dimethylpyridinium) tetrabromocuprate (DIMPY). In the gapless Tomonaga-Luttinger liquid phase we observe finite-temperature scaling of the $\mu^{+}$SR $1 / T_{1}$ relaxation rate which allows us to determine the Luttinger parameter $K$. We discuss the benefits and limitations of local probes compared with inelastic neutron scattering.
\end{abstract}

DOI: 10.1103/PhysRevB.95.020402

Quantum-critical states have attracted a great amount of theoretical and experimental interest since they exhibit universal behavior that is independent of the underlying microscopic Hamiltonian [1,2]. Of particular interest has been the universal scaling behavior of quantum critical phases that has so far been explored primarily using inelastic neutron scattering (INS) [3-7] even though many of the theoretical predictions actually concern local correlation functions which can also be explored using local probes such as nuclear magnetic resonance (NMR) [8,9] and muon-spin relaxation $\left(\mu^{+} \mathrm{SR}\right)$, both of which give access to an energy range effectively inaccessible to INS. $\mu^{+} \mathrm{SR}$ is established as a sensitive probe of magnetism and has been used to study quantum-critical spin dynamics in zero or "small" $(<1 \mathrm{~T})$ magnetic fields [10]. However, to the best of our knowledge, quantum-critical spin dynamics have never been explored using $\mu^{+} \mathrm{SR}$ in "high" $(>1 \mathrm{~T})$ applied fields, even though the majority of quantum-critical regions of interest is located at such magnetic fields. The reason for this were limitations in the high-field/low-temperature capabilities of existing $\mu^{+} \mathrm{SR}$ instruments and the difficulty of such experiments. The commissioning of the worldwide-unique HiFi instrument at ISIS, U.K. [11] now enables $\mu^{+}$SR to probe spin dynamics in longitudinal fields up to $5 \mathrm{~T}$ at $20 \mathrm{mK}$. The longitudinal (field parallel to initial muon spin) configuration is necessary for probing spin dynamics. Here, we report the observation of finite-temperature scaling of local spin correlations in the Tomonaga-Luttinger liquid phase of the strong-leg spin ladder bis(2,3-dimethylpyridinium) tetrabromocuprate (DIMPY) using $\mu^{+}$SR. This work demonstrates the feasibility of using high-longitudinal-field $\mu^{+}$SR to study quantum-critical spin dynamics.

Tomonaga-Luttinger liquid (TLL) theory provides a powerful, universal description of gapless interacting fermions in one dimension (1D), equivalent to the description that Landau Fermi liquid theory provides in three dimensions [1]. Within TLL theory the effects of interactions are contained

\footnotetext{
*Present address: Neutron Scattering and Magnetism, Laboratory for Solid State Physics, ETH Zürich, CH-8093 Zürich, Switzerland; jmoeller@phys.ethz.ch
}

within one single parameter, the Luttinger parameter $K$, where $K=1$ corresponds to noninteracting free fermions, $K<1$ describes repulsive interactions, and $K>1$ describes attractive interactions. This parameter $K$ universally defines all correlation functions regardless of the details of the interaction potential. We note that there is an additional parameter in the Luttinger model, the velocity $u$ of the excitations, which we are not sensitive to in this work. The experimental validation of the universal finite-temperature scaling relations, predicted by TLL theory [12], for spin correlations in $S=\frac{1}{2}$ Heisenberg chains using INS [3,4] was a particular triumph. However, Heisenberg chains, like most other experimental TLL model systems, are examples of a TLL with repulsive interactions $(K<1)[1,13]$ and until recently a TLL with attractive interactions was only known in certain quantum Hall edge states [14]. Spin ladders provide unique TLL model systems since the ratio of the rung and leg exchange determines the nature of the interactions between the spinless fermions in the system, with the prototypical strong-rung ladder $\mathrm{CuBr}_{4}\left(\mathrm{C}_{5} \mathrm{H}_{12} \mathrm{~N}\right)_{2}(\mathrm{BPCB})$ exhibiting repulsive behavior $[15,16]$ and the strong-leg ladder DIMPY exhibiting attractive interactions [7,8,17,18].

DIMPY is a two-leg spin-ladder system with a quantumdisordered (QD) singlet ground state with gap $\Delta_{0}=0.32(2)$ $\mathrm{meV}$ in zero field [19]. By virtue of the Zeeman effect this gap can be closed by an applied field $\mu_{0} H_{\mathrm{c} 1}=\Delta_{0} / g \mu_{B} \sim 2.85 \mathrm{~T}$ at a quantum-critical point (QCP) with a dynamic critical exponent $z=2$ (see Fig. 1) $[18,19]$. Above $H_{\mathrm{c} 1}$ and below the saturation field [21] $\mu_{0} H_{\mathrm{c} 2}=30 \mathrm{~T}$, the system is in the quantum-critical (QC) gapless $z=1 \mathrm{TLL}$ state. The dominant exchange interactions are $J_{\text {leg }}=1.42(6) \mathrm{meV}$ and $J_{\text {rung }}=$ 0.82 (2) $\mathrm{meV}$ along the ladder legs and rungs, respectively. In the gapless phase, three-dimensional long-range order (3D LRO) sets in at field-dependent critical temperatures of around $250 \mathrm{mK}$ due to weak interladder interactions. Using chain mean-field (MF) theory, interladder interactions were estimated [18] to be $n J_{\mathrm{MF}}^{\prime}=6.5 \mu \mathrm{eV}$, where $n$ is the number of relevant interaction pathways.

In a $\mu^{+} \mathrm{SR}$ experiment, spin-polarized positive muons are implanted into a sample. The experimentally measured quantity is the decay asymmetry $A(t)$ which is proportional to the spin polarization of the muon ensemble at any one time [22]. In this Rapid Communication we will concentrate 


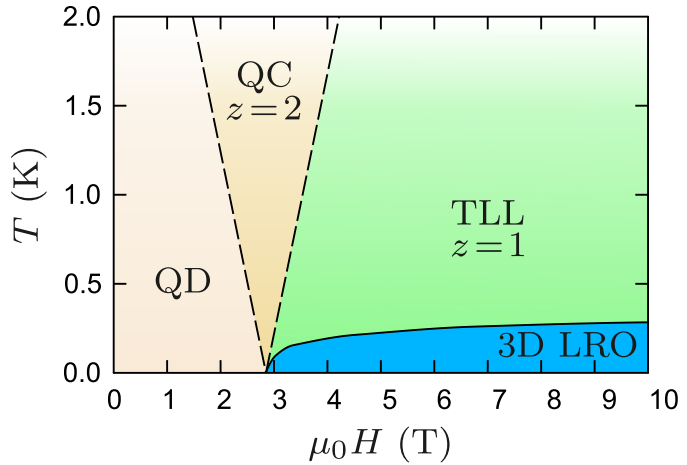

FIG. 1. Schematic phase diagram of DIMPY [8,18-20].

primarily on the longitudinal (spin-lattice) relaxation of the muon polarization $1 / T_{1}$. In direct analogy with NMR, $1 / T_{1}$ probes the local ( $q$-integrated) dynamic structure factor [see Eq. (5)] [23]

$$
\left.\frac{1}{T_{1}} \propto \int \sum_{\rho=x, y, z} \mathcal{S}^{\rho \rho}(\mathbf{q}, \omega)\right|_{\omega=\gamma_{\mu} B} d \mathbf{q},
$$

at the probing field $B$ and $\gamma_{\mu}$ is the muon gyromagnetic ratio. In general, $\mu^{+} \mathrm{SR}$ and NMR probe both longitudinal $\mathcal{S}^{z z}$ and transverse correlations $\mathcal{S}^{\perp \perp}$. Usually in a $\mu^{+}$SR experiment the magnetic coupling between the muon and sample is primarily of a dipolar nature, which leads to both transverse and longitudinal correlations being probed, while in NMR often the contact coupling is dominant, which implies that mainly transverse correlations are being probed [24]. However, the key property of the system studied here is that it is well known that only transverse correlations exist in the TLL phase at low energies $[16,17]$ which facilitates the study of their properties by $\mu^{+} \mathrm{SR}, \mathrm{NMR}$, and nonpolarized neutron scattering. For both NMR and $\mu^{+} \mathrm{SR}$ experiments the probing frequency corresponds to an energy scale of $\mu \mathrm{eV}$ in fields of a few tesla and so on the energy scale of the excitations in DIMPY and most other quantum magnets (which are on the meV scale) these effectively probe the local spin correlations as $\omega \rightarrow 0$, i.e., the long-time behavior of the local spin correlations. The probing energy scale is fixed for any given field.

Single crystal samples were grown by the method described in Ref. [25]. Initial $\mu^{+} \mathrm{SR}$ experiments were performed on a mosaic of single crystals, but the main $\mu^{+}$SR results presented here were obtained by crushing the crystals into a fine powder in order to cover a large area of the sample holder uniformly since the muon beam profile varies significantly as a function of magnetic field [11]. Due to the small $g$-factor anisotropy of the $\mathrm{Cu}^{2+}$ ions (expected $<10 \%$ ), the scaling functions in the TLL regime are effectively probed within a narrow range of fields, but since their dependence on field in the TLL phase is relatively weak, this effect is negligible. The powder sample was mounted with vacuum grease onto a silver sample holder which was attached to the cold finger of a dilution refrigerator at the HiFi instrument, ISIS, U.K. In high longitudinal fields, the relaxing asymmetry ( $A_{\text {rel }}$ below) is very small. The experiment therefore required high-statistics runs (40 million events) and careful attention to detector dead-time corrections by performing calibration measurements on a (a)

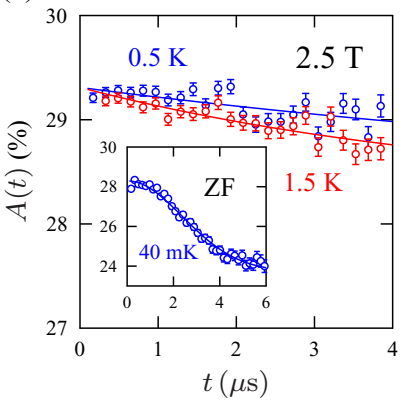

(b)

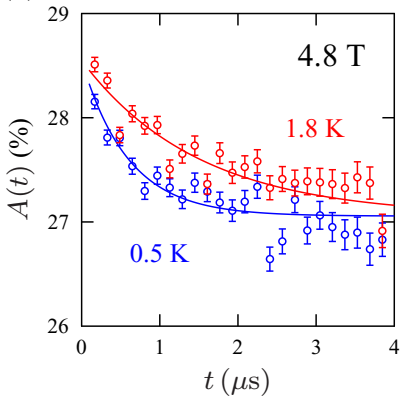

FIG. 2. Experimental muon decay asymmetry $A(t)$ (arbitrary offset). (a) Data in a longitudinal field $\mu_{0} H=2.5 \mathrm{~T}$ on the powder sample. Inset: Data measured in zero applied field on a mosaic of single crystals showing Gaussian relaxation due to nuclear moments. (b) Results in applied longitudinal field $\mu_{0} H=4.8 \mathrm{~T}$ (in the TLL phase).

silver backing plate at several fields covering the field range discussed here.

In Fig. 2 we show some of the $\mu^{+}$SR asymmetry data at different applied magnetic fields and temperatures. In high longitudinal fields it is not straightforward to calibrate the absolute scale of asymmetry, hence the data are shown with an arbitrary offset. In zero applied field the muon spectra display a temperature-independent Gaussian relaxation down to at least $40 \mathrm{mK}$, characteristic of a relaxation due to quasistatic nuclear moments without any contribution due to electronic moments. The zero-field data therefore demonstrate the absence of a phase transition in zero field in DIMPY down to $40 \mathrm{mK}$. At $2.5 \mathrm{~T}$ applied field, i.e., $H<H_{\mathrm{cl}}$, the relaxation is very weak at low temperatures, but there is a distinct increase in relaxation rate at higher temperatures. We expect any nuclear contribution to the muon relaxation to be fully quenched at this field and therefore we are only probing the electronic spin dynamics. Figure 3 shows $1 / T_{1}$ as a function of temperature at 2.5 and $4.8 \mathrm{~T}$ obtained by fitting the experimental asymmetry with

$$
A(t)=A_{\text {rel }} \exp \left(-t / T_{1}\right)+A_{\mathrm{nr}},
$$

where the relaxing asymmetry $A_{\text {rel }}$ was kept fixed and $A_{\mathrm{nr}}$ is a field-dependent nonrelaxing component [26]. At $\mu_{0} H=$ $2.5 \mathrm{~T}$, the system is still in the gapped singlet state and $1 / T_{1}$ is strongly suppressed. At temperatures $T \gg n J^{\prime}=75 \mathrm{mK}$ in the $z=21 \mathrm{D}$ quantum-critical regime, one expects [27] $1 / T_{1} \propto T^{-1 / 2}$. We observe an increase of $1 / T_{1}$ with increasing temperature that can be described phenomenologically by $1 / T_{1} \propto n(\Delta / T) T^{-1 / 2} \sim T^{+1 / 2}$ [where $n(\Delta / T)$ is the BoseEinstein occupation factor]. We note that this is similar to the behavior observed by NMR around the $z=2 \mathrm{QCP}$ in the spin-ladder system BPCB and the gapped quantum magnet $\mathrm{NiCl}_{2}-4 \mathrm{SC}\left(\mathrm{NH}_{2}\right)_{2}$ which was related to the effect of three-dimensional interactions [28].

As we increase the field to $\mu_{0} H=4.8 \mathrm{~T}$ and enter the TLL phase, the relaxation rate increases by approximately an order of magnitude. A temperature scan at constant field reveals a sharp rise of the relaxation rate with a peak around 225 $\mathrm{mK}$, followed by nonmonotonic behavior in an intermediate region between around 225 and $400 \mathrm{mK}$. Above $400 \mathrm{mK}$, $1 / T_{1}$ exhibits power-law behavior. We identify the peak in $1 / T_{1}$ around $225 \mathrm{mK}$ with a transition to long-range magnetic 


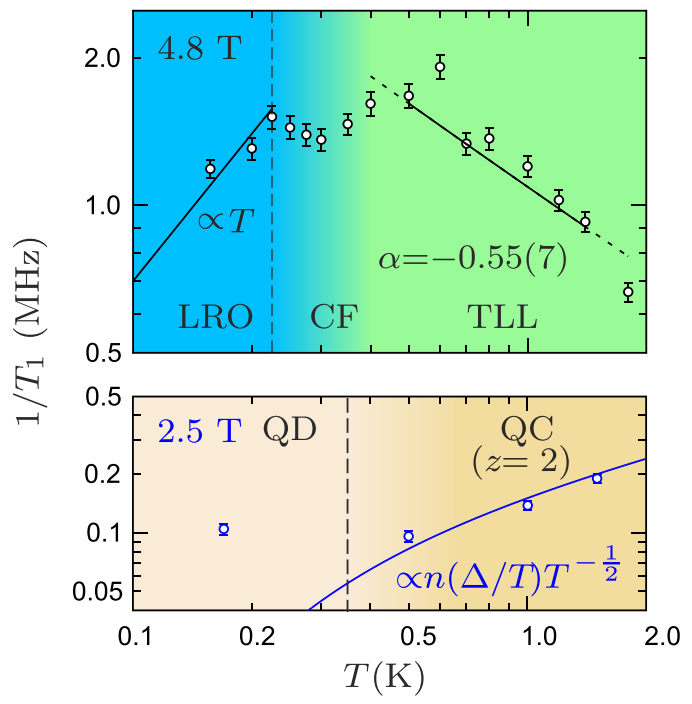

FIG. 3. Relaxation rate $1 / T_{1}$ at $\mu_{0} H=4.8 \mathrm{~T}$ (top) and $\mu_{0} H=$ $2.5 \mathrm{~T}$ (bottom). Top: The peak around $T=225 \mathrm{mK}$ indicates longrange ordering. Above the ordering transition, $1 / T_{1}$ is first dominated by critical fluctuations before entering a regime of universal scaling for $0.4 \mathrm{~K} \leqslant T \leqslant 2 \mathrm{~K}$. The solid dashed line is a fit to Eq. (3). Bottom: The dashed line indicates the approximate value of the gap for $g=$ 1.94 at $\mu_{0} H=2.5 \mathrm{~T}$.

order (LRO) previously observed $[8,18,20]$ in DIMPY around $250 \mathrm{mK}$ at $\mu_{0} H=5 \mathrm{~T}$. Below the ordering temperature our data are consistent with $1 / T_{1} \sim T$, which is expected due to the presence of a massless Goldstone mode [16]. However, the paucity of data in this region prevents definitive conclusions and further work is required to study the scaling behavior in this region. We note that the linear behavior of $1 / T_{1}$ in the 3D ordered state has not yet been observed by NMR [29]. The region immediately above the ordering temperature $T_{\mathrm{c}}$ is dominated by thermal critical fluctuations until power-law behavior sets in around $400 \mathrm{mK}$.

It has been shown that the dominant contribution to the local correlation function $\mathcal{S}(\omega)$ in a TLL at low energies is due to transverse correlations, which is a feature that is generic to spin ladders $[16,17]$. This greatly simplifies the present study as the presence of significant spectral weight in longitudinal correlations with different scaling properties at low energies would otherwise require longitudinal and transverse correlations to be studied separately. The transverse correlations take on an $\omega / T$ scaling form [2]

$$
\frac{1}{T_{1}} \propto \mathcal{S}^{\perp \perp}(\omega)=\left(k_{\mathrm{B}} T\right)^{\alpha} F\left(\hbar \omega / k_{\mathrm{B}} T\right),
$$

where $F\left(\hbar \omega / k_{\mathrm{B}} T\right)$ is a universal function [26] and $\alpha=$ $1 / 2 K-1, K$ being the Luttinger parameter $[16,17]$. Given the weak temperature dependence [26] of $F\left(\hbar \omega / k_{\mathrm{B}} T\right)$ in the $\omega \rightarrow$ 0 limit, the temperature dependence of $1 / T_{1}$ approximately follows a power law

$$
\frac{1}{T_{1}} \propto T^{\alpha}=T^{1 / 2 K-1}
$$

Let us now consider the appropriate fitting range for extracting the TLL parameter $K$. We argued that, at our probing

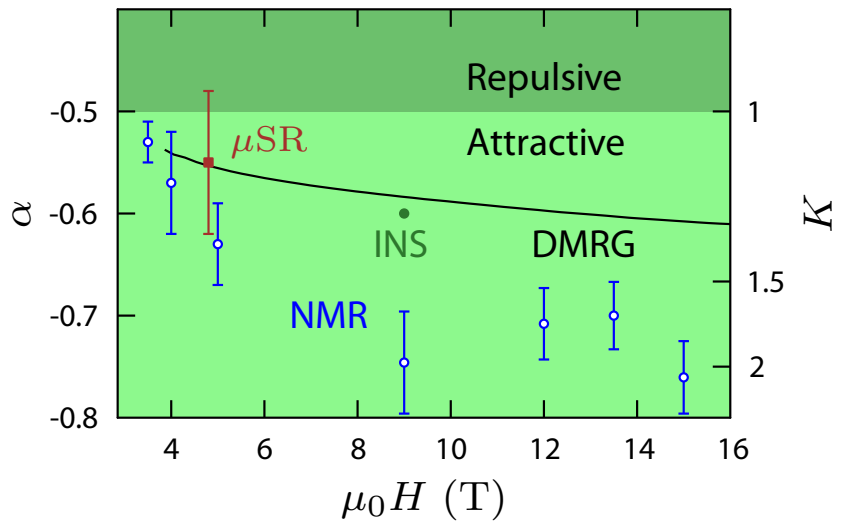

FIG. 4. Scaling exponent $\alpha$ and corresponding Luttinger parameter $K$ measured by ${ }^{1} \mathrm{H}$ NMR [8], INS [7], $\mu^{+}$SR (this work), and predicted by DMRG calculations [18].

field $\mu_{0} H=4.8 \mathrm{~T}$, thermal critical fluctuations are negligible above $\sim 0.4 \mathrm{~K}$. Furthermore, the TLL model requires (i) a linear dispersion relation and (ii) an infinitely deep Fermi sea. Requirement (i) is found to hold up to at least $1 \mathrm{meV}=11.6 \mathrm{~K}$ on the basis of previous INS data [7,30]. (ii) At $\mu_{0} H=$ $4.8 \mathrm{~T}$ the depth of the Fermi sea $\Delta_{\mathrm{F}}=g \mu_{\mathrm{B}} \mu_{0}\left(H-H_{\mathrm{c} 1}\right)=$ $0.22 \mathrm{meV}=2.5 \mathrm{~K}$ with $g=1.94$ and $\mu_{0} H_{\mathrm{c} 1}=2.85 \mathrm{~T}$ [18]. Hence a fitting range extending from 0.4 to $1.8 \mathrm{~K}$ is justified within the TLL framework. To further exclude any bias due to a particular fitting range, the data were fitted four times: over the full range $0.4-1.8 \mathrm{~K}$, excluding either end point, and excluding both end points. The final result is an average weighted by the inverse squared statistical errors. This method is similar to the shrinking-window method often used for extracting critical exponents. Following this procedure, fits to the full-scaling function Eq. (3) and the power-law approximation Eq. (4) yield $\alpha=$ $-0.55(7)[K=1.10(13)]$ and $\alpha=-0.62(5)[K=1.33(10)]$, respectively. The obtained TLL parameter $K>1$ indicates attractive interactions between the spinless fermions in the TLL.

Figure 4 shows a comparison of our $\mu^{+}$SR results for the Luttinger parameter with the previous experimental results based on NMR [8] and inelastic neutron scattering [7]. Also shown is the dependence of $K$ on the applied field based on density-matrix renormalization group (DMRG) calculations [18]. We note that the scaling function $F\left(\hbar \omega / k_{\mathrm{B}} T\right)$ does have a finite-temperature dependence on the $\mu^{+}$SR energy scales [26]. Hence we expect that parameters extracted by $\mu^{+}$SR from Eq. (3) to be more accurate than those extracted from the approximation in Eq. (4). This is consistent with the observation that the $\mu^{+}$SR estimates of $K$ using the former show better agreement with the DMRG calculations. NMR provides values of $\alpha$ that are lower, and, correspondingly, values of $K$ that are larger, than predicted by DMRG. Though only available at a single field, the $\mu^{+}$SR results seem to offer a somewhat better agreement with DMRG, although we acknowledge that the $\mu^{+}$SR and NMR error bars at 4.8 and $5 \mathrm{~T}$, respectively, overlap. Further to the discussion already presented in Ref. [8] about the quantitative disagreement at higher fields between NMR and DMRG, we believe that there are two contributing factors that have not been considered so far: (i) The NMR data were analyzed using the approximation 
in Eq. (4). Since $F\left(\hbar \omega / k_{\mathrm{B}} T\right)$ decreases as a function of temperature even in the applicable low-energy limit [26] but is assumed constant in Eq. (4), this leads to a fitted exponent in the power law that is somewhat too negative. We have digitized and reanalyzed some of the published NMR data [8] and find that this effect accounts for only approximately $1 \%-2 \%$ of the overestimate of $|\alpha|$. As the probing energy scales of NMR for a given field are lower than those of $\mu^{+} \mathrm{SR}$ by the ratio of muon and proton gyromagnetic ratios $\gamma_{\mu} / \gamma_{\mathrm{p}} \approx 3.18$, the temperature dependence of $F\left(\hbar \omega / k_{\mathrm{B}} T\right)$ is also weaker for NMR [26] by approximately this factor. Hence Eq. (4) is a better approximation when analyzing NMR data than for $\mu^{+}$SR data. (ii) The lower-energy scales probed by NMR lead to another problem: 3D interactions in DIMPY are accurately known $n J^{\prime}=6.5 \mu \mathrm{eV} \mathrm{[18].} \mathrm{The} \mathrm{proton} \mathrm{NMR} \mathrm{probing} \mathrm{energy}$ scale at $\mu_{0} H=5 \mathrm{~T}$ is $\hbar \omega=0.88 \mu \mathrm{eV}$ compared to $\hbar \omega=$ $2.7 \mu \mathrm{eV}$ for $\mu^{+} \mathrm{SR}$ at $\mu_{0} H=4.8 \mathrm{~T}$. Therefore, any perturbing effects due to $3 \mathrm{D}$ interactions will be more pronounced at any given field for NMR than for $\mu^{+} \mathrm{SR}$.

Finally, in an inelastic neutron scattering experiment, the dynamic structure factor

$$
\mathcal{S}^{\rho \rho}(\mathbf{q}, \omega)=\int_{-\infty}^{\infty}\left\langle S^{\rho}(\mathbf{0}, 0) S^{\rho}(\mathbf{r}, t)\right\rangle \exp [i(\omega t-\mathbf{q} \cdot \mathbf{r})] d \mathbf{r} d t
$$

can be probed as a function of energy and momentum transfer $(\rho=x, y, z)$. INS therefore allows a certain region of interest in energy-momentum space to be selected and, in particular, it provides access to energy as an additional independent parameter. By comparing the experimental INS spectra with DMRG calculations, it was possible to identify the parts of the spectrum where the transverse correlations described in Eq. (3) dominate [7]. By using energy transfer and temperature as independent parameters it was possible to probe universal scaling over more than two decades in $\hbar \omega / k_{\mathrm{B}} T$, leading to an excellent agreement with DMRG calculations. Scaling was also observed by INS within the LRO phase $T<T_{\mathrm{c}}$ since the considered energy scales $0.1 \mathrm{meV}<\hbar \omega<0.5 \mathrm{meV}$ are well above the energy scale of the $3 \mathrm{D}$ interactions. The exact value of the determined Luttinger parameter at $\mu_{0} H=9 \mathrm{~T}$ depends on the details of the analysis with values in the range: $K=1.25$ in Ref. [7], $K=1.2(1)$ and $K=1.19$ (2) in Ref. [31].

In Fig. 5 we compare the different scales in $\hbar \omega / k_{\mathrm{B}} T$ probed by $\mu^{+}$SR, NMR, and INS. $\mu^{+}$SR fills a gap that is inaccessible to both NMR and INS. In NMR similar energy scales could only be achieved at much higher fields, implying that a

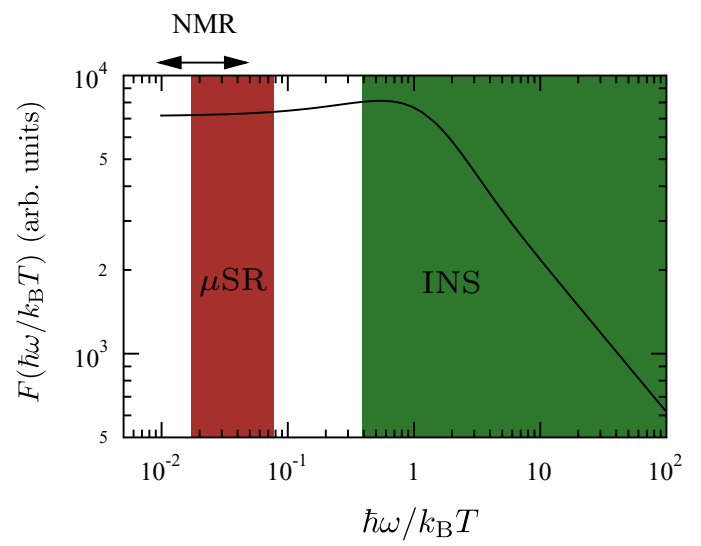

FIG. 5. Comparison of the energy scales probed by the $\mu^{+} \mathrm{SR}$ experiment reported in this work, the NMR experiment [8], and the INS experiment [7]. The solid line gives the scaling function [26] $F\left(\hbar \omega / k_{\mathrm{B}} T\right)$ for the local dynamic structure factor with $K=1.1$.

different region of the phase diagram is being investigated. In INS such energy scales are practically inaccessible even with state-of-the-art cold neutron spectrometers.

In conclusion, using an exceptionally clean and wellcharacterized model system of a Tomonaga-Luttinger liquid, we have demonstrated that high-field $\mu^{+}$SR can be used to probe quantum-critical spin dynamics in a magnetic field range that is of great experimental interest in many topical materials. $\mu^{+}$SR fills a gap in energy scales that is inaccessible to established techniques such as NMR and INS. Model systems where $\mu^{+}$SR would prove particularly useful are those where INS is difficult to perform or universal behavior at the lowest-energy scales is of interest.

J.S.M. is grateful to Professor Andrey Zheludev, Dr. Martin Klanjšek, Dr. Matthias Thede, and Dr. Kirill Povarov for many helpful discussions. We thank Dr. James Lord for helpful comments concerning the detector dead-time corrections. J.S.M. is grateful for support by the ETH Zurich Postdoctoral Fellowship Program which has received funding from the European Union's Seventh Framework Programme for research, technological development, and demonstration under Grant Agreement No. 246543. This project was supported by EPSRC (U.K.). Part of this work was carried out at the STFC ISIS facility and we are grateful for the generous provision of beam time.
[1] T. Giamarchi, Quantum Physics in One Dimension (Oxford University Press, Oxford, U.K., 2003).

[2] S. Sachdev, Quantum Phase Transitions (Cambridge University Press, Cambridge, U.K., 1999).

[3] D. C. Dender, Spin dynamics in the quasi-one-dimensional $S=$ $1 / 2$ Heisenberg antiferromagnet copper benzoate, Ph.D. thesis, Johns Hopkins University, 1997.

[4] B. Lake, D. A. Tennant, C. D. Frost, and S. E. Nagler, Nat. Mater. 4, 329 (2005).

[5] M. Hälg, D. Hüvonen, N. P. Butch, F. Demmel, and A. Zheludev, Phys. Rev. B 92, 104416 (2015)
[6] M. Hälg, D. Hüvonen, T. Guidi, D. L. Quintero-Castro, M. Boehm, L. P. Regnault, M. Hagiwara, and A. Zheludev, Phys. Rev. B 92, 014412 (2015).

[7] K. Y. Povarov, D. Schmidiger, N. Reynolds, R. Bewley, and A. Zheludev, Phys. Rev. B 91, 020406 (2015).

[8] M. Jeong, H. Mayaffre, C. Berthier, D. Schmidiger, A. Zheludev, and M. Horvatić, Phys. Rev. Lett. 111, 106404 (2013).

[9] A. W. Kinross, M. Fu, T. J. Munsie, H. A. Dabkowska, G. M. Luke, S. Sachdev, and T. Imai, Phys. Rev. X 4, 031008 (2014).

[10] See, for example, Ref. [32]. 
[11] J. S. Lord, I. McKenzie, P. J. Baker, S. J. Blundell, S. P. Cottrell, S. R. Giblin, J. Good, A. D. Hillier, B. H. Holsman, P. J. C. King, T. Lancaster, R. Mitchell, J. B. Nightingale, M. Owczarkowski, S. Poli, F. L. Pratt, N. J. Rhodes, R. Scheuermann, and Z. Salman, Rev. Sci. Instrum. 82, 073904 (2011).

[12] F. D. M. Haldane, Phys. Rev. Lett. 45, 1358 (1980).

[13] H. J. Schulz, Phys. Rev. B 34, 6372 (1986).

[14] M. Grayson, L. Steinke, D. Schuh, M. Bichler, L. Hoeppel, J. Smet, K. v. Klitzing, D. K. Maude, and G. Abstreiter, Phys. Rev. B 76, 201304 (2007).

[15] M. Klanjšek, H. Mayaffre, C. Berthier, M. Horvatić, B. Chiari, O. Piovesana, P. Bouillot, C. Kollath, E. Orignac, R. Citro, and T. Giamarchi, Phys. Rev. Lett. 101, 137207 (2008).

[16] T. Giamarchi and A. M. Tsvelik, Phys. Rev. B 59, 11398 (1999).

[17] T. Hikihara and A. Furusaki, Phys. Rev. B 63, 134438 (2001).

[18] D. Schmidiger, P. Bouillot, S. Mühlbauer, S. Gvasaliya, C. Kollath, T. Giamarchi, and A. Zheludev, Phys. Rev. Lett. 108, 167201 (2012).

[19] T. Hong, Y. H. Kim, C. Hotta, Y. Takano, G. Tremelling, M. M. Turnbull, C. P. Landee, H.-J. Kang, N. B. Christensen, K. Lefmann, K. P. Schmidt, G. S. Uhrig, and C. Broholm, Phys. Rev. Lett. 105, 137207 (2010).

[20] K. Ninios, T. Hong, T. Manabe, C. Hotta, S. N. Herringer, M. M. Turnbull, C. P. Landee, Y. Takano, and H. B. Chan, Phys. Rev. Lett. 108, 097201 (2012).

[21] J. L. White, C. Lee, O. Gunaydin-Sen, L. C. Tung, H. M. Christen, Y. J. Wang, M. M. Turnbull, C. P. Landee,
R. D. McDonald, S. A. Crooker, J. Singleton, M.-H. Whangbo, and J. L. Musfeldt, Phys. Rev. B 81, 052407 (2010).

[22] S. J. Blundell, Contemp. Phys. 40, 175 (1999).

[23] T. Moriya, Prog. Theor. Phys. 16, 23 (1956).

[24] A. Yaouanc and P. D. D. Réotier, Muon Rotation, Relaxation, and Resonance (Oxford University Press, Oxford, U.K., 2011).

[25] A. Shapiro, C. P. Landee, M. M. Turnbull, J. Jornet, M. Deumal, J. J. Novoa, M. A. Robb, and W. Lewis, J. Am. Chem. Soc. 129, 952 (2007).

[26] See Supplemental Material at http://link.aps.org/supplemental/ 10.1103/PhysRevB.95.020402 for further details on the scaling function, the field-dependent muon data, and the data shown in the figures of this Rapid Communication.

[27] E. Orignac, R. Citro, and T. Giamarchi, Phys. Rev. B 75, 140403 (2007).

[28] S. Mukhopadhyay, M. Klanjšek, M. S. Grbić, R. Blinder, H. Mayaffre, C. Berthier, M. Horvatić, M. A. Continentino, A. Paduan-Filho, B. Chiari, and O. Piovesana, Phys. Rev. Lett. 109, 177206 (2012).

[29] M. Klanjšek (private communication).

[30] D. Schmidiger, P. Bouillot, T. Guidi, R. Bewley, C. Kollath, T. Giamarchi, and A. Zheludev, Phys. Rev. Lett. 111, 107202 (2013).

[31] D. Schmidiger, Physics of a strong-leg quantum spin ladder, Ph.D. thesis, ETH Zurich, 2014.

[32] F. L. Pratt, P. J. Baker, S. J. Blundell, T. Lancaster, S. OhiraKawamura, C. Baines, Y. Shimizu, K. Kanoda, I. Watanabe, and G. Saito, Nature (London) 471, 612 (2011). 